\title{
An unusual Guillain-Barré Syndrome mimic: a case of Spinal ADEM and review of the literature
}

\author{
Homayoun Roshanisefat ${ }^{1 *}$, Bimma Henderson ${ }^{2}$ and Rayomand Press \\ ${ }^{1}$ Department of Neurology, Karolinska University Hospital, Stockholm, Sweden \\ ${ }^{2}$ Department of Radiology, Karolinska University Hospital, Stockholm, Sweden
}

\begin{abstract}
Background: Acute disseminated encephalomyelitis (ADEM) is one the most common inflammatory demyelinating brain disorders in children and adolescence and it is considered to constitute a third of all encephalitis. To date only few cases of ADEM confined to the spinal cord have been reported in the literature, though this may in part be due to the fact that patients with suspicion of encephalitis are usually not worked-up with a spinal MRI. However, the pathogenesis of spinal ADEM is similar to that of brain ADEM, including a similar relationship to antecedent infections and vaccinations, making it necessary for the clinician to recognize the fact that some cases of ADEM may be restricted merely to the spinal cord.

Case presentation: Here, we describe a 45 year old female with a post-infectious sub-acute ascending sensorymotor disturbance eventually affecting all four limbs, hyporeflexia and a long-stretched multilevel intraspinal inflammatory lesion suggesting spinal ADEM. Brain magnetic resonance imaging (MRI) was normal, though the spinal MRI revealed intraspinal hyperintensity contrast-enhancing lesions in the entire spinal cord and the cauda equina. The differential diagnosis based on clinical signs, results of cerebrospinal fluid analysis and imaging included Guillain-Barré syndrome (GBS), Chronic Inflammatory Demyelinating Polyradiculoneuropathy (CIDP), Chronic lymphocytic inflammation with pontine perivascular enhancement responsive to steroids (CLIPPERS), Multiple Sclerosis, Neuromyelitis Optica and spinal cord malignancy. A good clinical response as well as complete radiological resolution was achieved after high-dose corticosteroid therapy and six plasma exchanges leading to an improvement from wheel-chair dependence due to paraparesis, to an ability to walk outdoors without need for any walking aids, upon last follow-up eleven months after onset of neurological symptoms.

Conclusions: This case of isolated spinal ADEM presented here had similar CSF findings and clinical, as a well as radiological outcome after immunomodulatory treatment as cerebral ADEM. Further studies are needed to elucidate if spinal ADEM is often misdiagnosed as mere transverse myelitis, and to estimate the long term outcome and mortality rate of spinal cord ADEM.
\end{abstract}

\section{Introduction}

The non-neoplastic spinal cord diseases are heterogeneous with regards to the variety of underlying pathogenic mechanisms such as autoimmunity, infectious, toxic, nutritional, vascular and paraneoplastic conditions. Here we present an acute post-infectious inflammatory disorder of the spinal cord in a 45 year old Caucasian healthy female with no family or own history of neurological disease. This case was referred to the emergency room of neurological services at Karolinska University Hospital in the spring of 2016 for evaluation of a subacute progressive impairment of gait with an onset earlier during the year. The initial suggested diagnosis was Guillain-Barré syndrome (GBS), though the neurophysiological examination turned out to be normal. The various differential diagnosis challenge of this case began after the unexpected spinal MRI results.

\section{Case report}

The patient and her two children had onset of gastroenteritis (GE) with diarrhea and vomiting eight weeks prior to the initial presentation at our emergency room. The GE had lasted for 24 hours. The day after onset of GE, the patient began to feel a burning sensation on the right side of the scalp as well as slight pain in the lower back. Two days later, she sought a general practitioner at her nearby community medical center, due to the lumbar discomfort and numbness in the feet and hands. Infection parameters and antibodies to Lyme disease in blood were normal. Due to increase in the intensity of the sensory symptoms during the following four weeks, a brain MRI was planned for to exclude multiple sclerosis (MS). On day 33 after onset of the initial neurological symptoms, she experienced onset of weakness in the legs and an increase in level of numbness in the hands and feet. The weakness and numbness were most pronounced in the right foot but was also present in both lower legs. The numbness then spread to the thighs and the dorsal side of the hands and elbows. Eight weeks after onset of neurological symptoms the patient was unable to walk without aid due to weakness of the right leg. She also experienced pinand-needle pain in the right arm and leg and felt weak in both legs. She could not walk more than 2-5 meters. She also had a dull pain sensation around the pelvis and lumbar spine, which radiated to the right thigh, where she also felt a burning sensation. At this stage, there

Correspondence to: Homayoun Roshanisefat, Department of Neurology, Karolinska University Hospital, Huddinge, 14186 Stockholm, Sweden, Tel: +46 858578279; E-mail: homayoun.roshanisefat@ki.se

Key words: Biomarkers, CSF, MRI, Acute disseminated encephalomyelitis (ADEM), Multiple Sclerosis (MS), Transverse Myelitis (TM), Guillain-Barré Syndrome (GBS), Chronic Inflammatory Demyelinating Polyradiculoneuropathy (CIDP), Chronic lymphocytic inflammation with pontine perivascular enhancement responsive to steroids (CLIPPERS), Neuromyelitis Optica (NMO), plasma exchange

Received: November 25, 2016; Accepted: December 19, 2016; Published: December 22, 2016 
was no numbness in the genital area, or disturbance of micturition and defecation. No fever was reported.

\section{Findings upon arrival at Karolinska University Hospital, eight weeks after symptom onset}

General examination: Cognition, including language and orientation were normal. The patient was afebrile, was normotensive and had a blood saturation of $98 \%$. The abdomen was assessed as soft and non-tender. Pudendus, pubis and perianal sensibility, as well as sphincter tonus were normal. Lymph-nodes palpation was with no remarks. The CRP, SR and leukocyte count were normal.

Neurological examination: The gait was unsteady. Walking on toes was possible though with great difficulty; walking on heels was normal. The patellar and achilles reflexes were very weak bilaterally. Babinski's sign was negative. Plantar and dorsal flexion and straight leg extension were intact. A follow-up of muscle strength is shown in table 1 . Right sided patchy hypoesthesia was detected in both hands, reaching the elbows and in lower extremities symmetrically up to the mid-thigh. Reduced sensation of vibration in the legs bilaterally, though more pronounced in right side and impaired proprioception in the right leg and foot was seen. Squatting was impaired.

The complete neurophysiological tests (electroneurography) were normal, but the MRI showed a multilevel inflammatory long lesion in the spinal cord stretching from $\mathrm{C} 1$ to conus medullaris (Figures 1a-d), so iv methyl-prednisolone was administered leading to some improvement of the sensory symptoms. Ten days later the patient was transferred to a multidisciplinary rehabilitation unit, but was sent back to the emergency room at Karolinska University Hospital three days later (10 weeks after the onset of neurological symptoms), due to further deterioration of both motor and sensory function.

Findings upon arrival at the emergency room at Karolinska University Hospital (return visit), 10 weeks after symptom onset

At the second examination upon return to Karolinska University Hospital the patient was wheelchair-bound and described some delay in the capability to initiate defecation and micturition. The reflexes in the upper limbs were weaker compared to the prior examination eight weeks after onset of neurological symptoms. There was a weakness in the lumbo-sacral muscles, including the Psoas major, Iliacus and Iliocostalis lumborum muscles. The patellar reflexes and Achilles were now absent. The patient could stand up without support, but unsteadily due to paresis in right leg. Muscle strength was $2 / 5$ the right leg, $4 / 5$ in

Table 1. Clinical course over time and response to immunomodulatory treatments given:

\begin{tabular}{|c|c|c|c|c|c|}
\hline & Test 1 & Test 2* & Test 3** & Test 4*** & Test 5*** \\
\hline $\begin{array}{l}\text { Weeks after onset of } \\
\text { symptoms }\end{array}$ & 8 & 12 & 16 & 25 & 34 \\
\hline Muscle strength (MRC) & & & & & \\
\hline UEBilateral (R):(L) & $4 / 5: 5 / 5$ & $5 / 5: 5 / 5$ & $5 / 5: 5 / 5$ & $5 / 5: 5 / 5$ & $5 / 5: 5 / 5$ \\
\hline Right hip & $1 / 5$ & $3 / 5$ & $4 / 5$ & $5 / 5$ & $5 / 5$ \\
\hline Left hip & $3 / 5$ & $4 / 5$ & $4 / 5$ & $5 / 5$ & $5 / 5$ \\
\hline Right knee & $2 / 5$ & $3 / 5$ & $4 / 5$ & $5 / 5$ & $5 / 5$ \\
\hline Left knee & $3 / 5$ & $4 / 5$ & $4 / 5$ & $5 / 5$ & $5 / 5$ \\
\hline Right ankle & $0 / 5$ & $2 / 5$ & $4 / 5$ & $4 / 5$ & $5 / 5$ \\
\hline Left ankle & $3 / 5$ & $4 / 5$ & $4 / 5$ & $5 / 5$ & $5 / 5$ \\
\hline
\end{tabular}

*After methyl-prednisolone, **after PE, ***According to evaluation of physiotherapist; UE: Upper-extremity (Hand, forearm and upper arm), R: right, L: left; MRC: Medical Research Council Scale ${ }^{27}$ the left leg, 4/5 in the right hand and 5/5 in the left hand/arm (Table 1).

There was no Lhermitte's sign. A clear loss of sensation for temperature, pressure and vibration were evident under the C1-level, though most pronounced distally in the right leg and arm and the patient was completely unable to discriminate between sharp, dull and or unable to feel light touch distally in the right arm and leg. The anal sphincter was weak and the sensation was reduced in the anal canal. No intention tremor, dysmetria, or ataxia was seen. No herpes vesicles or any skin rash was observed.

\section{Neurophysiological examination, performed during the $8^{\text {th }}$ week after onset symptoms}

The motor and sensory conduction velocities and amplitudes, distal latencies and F-responses were normal. Evoked potentials including visual (VEP) was normal, but the somatosensory evoked potentials (SSEP) showed a slight delay in tibial nerves bilaterally, with normal SSEP latencies upon stimulation of median nerves.

\section{Radiology at first Presentation}

Radiographic skeletal X-ray of vertebrate was normal.

The first MRI of the brain during the $8^{\text {th }}$ week (fifty two days) after symptom onset was normal, but the MRI of the spinal cord showed a long-stretched signal change between $\mathrm{C} 1$ and conus medullaris on T2-sequences (Figure 1a). T1-sequences showed bilateral medullar gadolinium contrast enhancement of the entire spinal cord at all levels, i.e. $\mathrm{C} 1$ to conus medullaris (Figures $1 \mathrm{c}$ and $1 \mathrm{~d}$ ). The gadolinium enhancing lesions had indistinct borders in the gray and white matter on T1-whighted images (Figure 1e). The extensive lesions were localized bilaterally in the lateral, dorsal and ventral horns, spanning the entire cervical cord from $\mathrm{C} 1$ to the conus medullaris, though with a posterolaterally right-sided dominance (Figures 1e and 1i). In addition, leptomeningeal enhancement was seen at the Th 2-3 level. Swelling of the spinal cord was evident at the C5-C7 segments (Figure 1c, white arrows). Mechanical compression of the spinal cord, disc or spinal metastasis, neoplastic as well as paraneoplastic myelopathy were excluded radiologically.

CT of the thorax/abdomen was normal and did hence show no signs of sarcoidosis, lymphoma, mycobacterial infection or malignancy.

\section{Laboratory findings}

\section{Blood}

The CRP, white and red blood cells, electrolytes (Sodium, Potassium and Calcium), the $\mathrm{B}_{12}$ level, liver parameters, Creatinine and urine dipstick were within normal limits.

Blood-protein fraction did show no signs of M-component. Vasculitis blood panel, including ANA and ANCA and Complement levels were with no remarks. Serum paraneoplastic-antibodies, aquaporin-4-antibody, cardiolipin, a wide range of parameters for systemic disease such as lupus erythematosus (SLE) were normal.

\section{The cerebrospinal fluid (CSF) at presentation and follow-up}

The CSF assay was performed twice over a two week interval. The first lumbar puncture (LP1) performed eight weeks from onset of neurological symptoms showed a slight increase in the CSF: plasma albumin index to $9.4 \times 10^{-3}$ (normal reference $<7$ ) and a mononuclear pleocytosis with 33,8 mononuclear cells per $\mu$. The second CSF analysis (LP2) performed three days after the methyl-prednisolone 

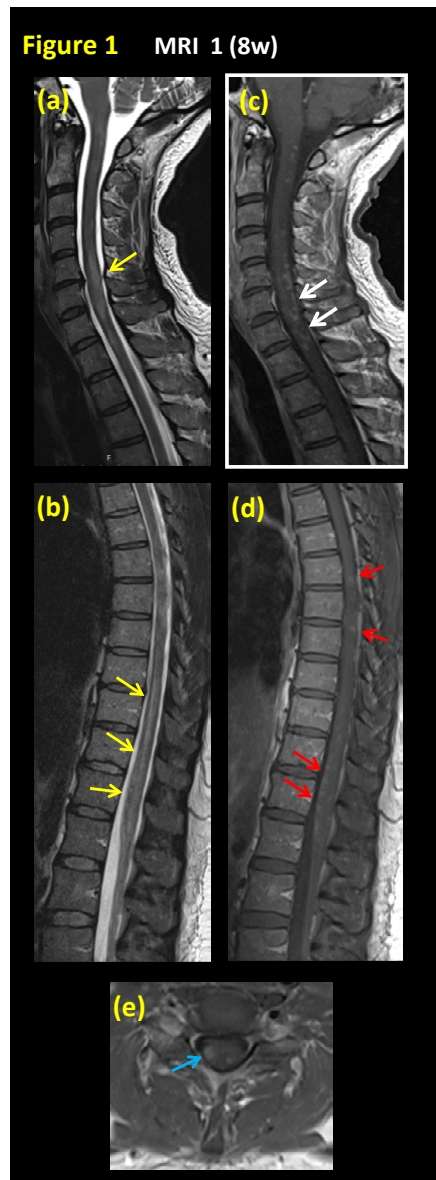
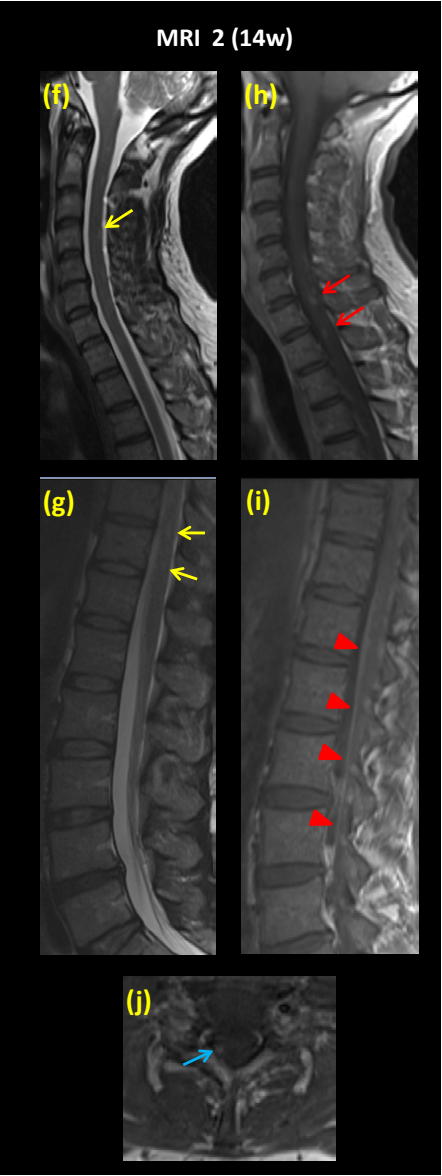
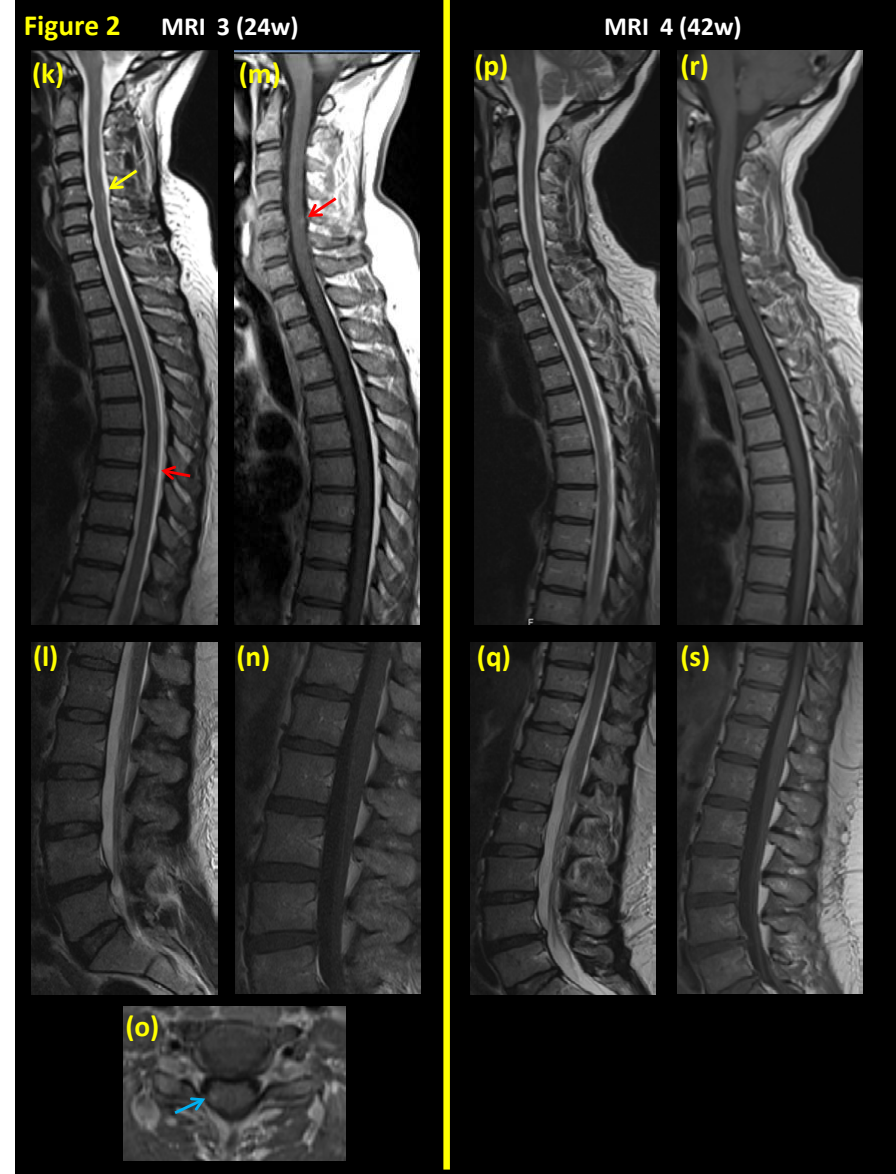

Figures 1 and 2. SpinalMRI series during the course of disease. MRI no. 1 (a-e), eight weeks after onset of neurological symptoms; MRI no. 2 (f-j ), fourteen weeks after onset of symptoms; and MRI no. 3 (k-o), twenty four weeks post-onset and finally MRI no. 4 (p-s), forty two weeks post-onset.

Figures 1-a and b demonstratelong intraspinal lesions of hyperintensity (yellow arrows) on sagittal T2WI. There is also a slight swelling of the cervical medulla.

Figures 1-c and dshow multiple gadolinium contrast-enhancing lesions within the spinal cord at levels (C1 - conus medullaris) on T1WI+C (white arrows).

Figure 1e is a transverse section at the cervical level (C7) showing the lesion to be predominantly localized posterolaterally in the spinal cord, mainly on the right side (blue arrows).

Figure 1i is a sagittal T1WI+C image showing contrast enhancement of the caudaequina and spinal roots as showed by red arrowheads.

Figures 1e, j and Figure 2oare transverse T1WI+C images after gadolinium infusion, showing diminishing lesion size as well as extent of contrast enhancement (blue arrows) following immunomodulatory treatment.

Figures 2k-n show T2WI with only small areas of focal intramedullary hyperintenisty(yellow arrows) andon T1WI+C there is only a residual of contrast enhancement in the cervical spinal cord at level C5 (red arrow).

Figures 2p-s show complete resolution of intraspinal lesions without any remaining focal areas of hyperintensity or contrast enhancement.

Intravenous methyl-prednisolone and Plasma Exchange was administered between the MRIs 1 and 2. Oral steroids were administered between MRIs 2 and 3.

infusion (14 days after LP1) showed 21 mononuclear cells per $\mu$ l. The CSF neurofilament light (NFL) chain was $9560 \mathrm{ng} / \mathrm{L}$ (normal reference $<870)$ at the 1 st CSF examination (LP1). There was no increased immunoglobulin (Ig) G index or oligoclonal bands (OCB) in the CSF. At LP2, the CSF-lactate was $2.4 \mathrm{mmol} / \mathrm{L}$ (normal reference $0.5-2.3$ $\mathrm{mmol} / \mathrm{L}$ ). Angiotensin converting enzyme (ACE) levels in blood and CSF were normal.

CSF and blood polymerase chain reaction (PCR) for virology profile (HSV, HBV, HIV, HTLV-1, CMIA, Entrovirus, HSV-1, HSV2, and HZV) and enzyme-linked immunosorbent assay (ELISA) test for Lyme disease, Syphilis, chlamydia, Mycoplasma pneumonia and Parapertusis were negative.

Further analysis showed no morphologically atypical CSF cell malignancy, signs of intrathecal lymphoma or increase in levels of paraneoplastic antibodies in the CSF and blood (Table 2).

\section{Differential diagnosis}

The first diagnosis which was considered based on the postinfectious nature of the disease, the ascending paralysis in the legs and the emerging hyporeflexia was GBS. The suspicion of GBS was however neither supported by the duration of the progressive phase of disease (which exceeded 4 weeks), results of the neurophysiological tests, nor the subsequent spinal MRI results. The alternative diagnosis of chronic inflammatory demyelinating polyradiculoneuropathy (CIDP) was also entertained, but was neither supported by the results of the neurophysiological tests (showing no electrophysiological signs of peripheral nerve affection), nor by the MRI showing signs of a predominantly central neuroinflammatory disorder.

The next condition to be considered was a myelopathy due to the spinal MRI findings. The initial radiological diagnosis suggested by the neuroradiologists after review of the first MRI were CLIPPERS and lymphoma (Figures 1a-1d). However, based on the clinical signs, radiological and CSF findings it was deemed necessary to consider an 
Table 2. CSF findings in inflammatory and malignant diseases of the spinal cord.

\begin{tabular}{|l|c|c|c|c|}
\hline & ADEM & CLIPPERS & MS & NMO \\
\hline Cells, mononuclear & Increased [4] & Increased [24] & Common [34] & Mildly Increased* [35] \\
\hline CSF-protein & Increased [34] & Increased [26] & Occasionally [37] & Increased [34] \\
\hline CSF-Albumin-index & Increased [39] & NR & Normal & Increased [40] \\
\hline IgG-index & Normal & NR & Increased & Negative [40] \\
\hline Oligoclonal bands & Negative & Often Positive & Positive & No [40] \\
\hline Neurofilament light & NR & NR & Variable & Increased [43] \\
\hline CXCL13 & NR & NR & Increased & Increased [45] \\
\hline Viral PCR** & NR & NR & Negative & Negative \\
\hline Bacterial PCR & NR & NR & Negative & Negative \\
\hline Aquaporin-4 antibody & Negative & NR & Negative & Parionally [38] \\
\hline Lactate & Normal & NR & Increased & Neasitive [48] \\
\hline
\end{tabular}

NR: Not Reported; TM: Transverse Myelitis; NMO: Neuromyelitis optica; ADEM: Acute disseminated encephalomyelitis; MS: Multiple Sclerosis

CLIPPERS: Chronic Lymphocytic Inflammation With Pontine Perivascular Enhancement Responsive to Steroids; *Mixed mono and poly in 15-30\% of cases.

**Virology profile: HSV, HBV, HIV, HTLV-1, CMIA, Enterovirus, HSV-1, HSV-2, and HZV

even wider range of differential diagnoses associated with acute myelitis, i.e. Multiple sclerosis (MS), Neuromyelitis optica (NMO), Acute disseminated encephalomyelitis (ADEM), idiopathic acute transverse myelitis (IATM), infectious myelitis, systemic lupus erythematosus and neurosarcoidosis (Table 2).

In the $14^{\text {th }}$ week after onset of neurological symptoms, the results of the second MRI (Figures 1f-1i) which was performed after treatment with iv methyl-prednisolone and Plasma Exchange (PE) two weeks after the first MRI, helped narrow down the list of differential diagnosis, as this MRI (Figures 1f-1i) showed a reduction in the level of the confluent scattered hyperintense lesions in the greater part of the spinal cord in the $\mathrm{C} 1$ - conus medullaris levels and as well regression of the swelling of the spinal cord, C5-C7, compared with the first MRI (Figures 1a-1e). Surprisingly, the second MRI (Figures 1f-1i) revealed gadolinium enhancement even of the cauda equina corresponding to the ventral sacral roots (Figure 1i, red arrows), and the transverse section showed no sign of medullary lesions involving the central canal (Figure 1f). The results of the second MRI challenged the previous suspicion of an isolated myelitis, since the MRI now showed evidence of a combined CNS and peripheral nervous system (PNS) inflammation (i.e. a myeloradiculitis) not expected in MS/NMO, but reported rarely in ADEM [1]. Due to this unexpected radiological finding, the MRI was reviewed by a second group of neuroradiologists who did concur on the finding of gadolinium enhancement of the cauda equina, did not regard Lymphoma and CLIPPERS to be likely, but rather suggested that a systemic vasculitic disorder with secondary neurological manifestations be considered (Figure 1e).

\section{Administration of treatments and results}

Intravenous Steroid Treatment: Based on the first spinal-MRI findings (Figures 1a-1e), the patient was treated with intravenous methyl-prednisolone during the $8^{\text {th }}$ week after onset of neurological symptoms, at a total dose of $20 \mathrm{mg} / \mathrm{kg}, 1.500 \mathrm{mg} / \mathrm{d}$ for 3 days, leading to a partial improvement of sensory symptoms. However, the patient deteriorated with regards to walking ability ten days after the termination of the iv methyl-prednisolone treatment and was returned to Karolinska University Hospital from the rehabilitation hospital.

\section{Plasma Exchange (PE)}

During the $10^{\text {th }}$ week after onset of neurological symptoms (11 days after the first visit to the Department of Neurology; 10 days after the first corticosteroid treatment), a course of six treatments with PE was given. The plasma was replaced with $1,500 \mathrm{ml}$ of $4 \%$ albumin and
1,500 ml Octaplas (Pooled Human Plasma).

\section{Oral steroids}

\section{a) Deltison}

T. Prednisone (Deltison) $50 \mathrm{mg}, 20$ tablets /day for 3 days was administered one month after the PE.

\section{b) Prednisolone}

Treatment with T. Prednisolone $10 \mathrm{mg}$ per day was hence initiated 3 days after the termination of the oral Prednisone (Deltison) treatment and continued for 3 weeks, followed by a slow taper and discontinuation during a further eight weeks. The total length of treatment period with Prednisolone was hence 11 weeks.

\section{Outcome}

The initial methyl-prenisolone therapy was not very efficacious, though the rate of decline was temporary reduced. The second MRI performed 14 weeks after the onset of neurological symptoms, showed only a partial improvement in the level of gadolinium enhancement and edema of the spinal lesion (Figures 1f-1i), which led to the decision to treat the patient with a course of pulsed oral high dose steroids.

The third MRI twenty four weeks after onset of neurological symptoms (10 weeks after the second MRI), showed a more or less complete resolution of the intraspinal inflammatory lesion, though a very slight residual gadolinium enhancement was seen in the cervical spinal cord segments C5-C7 (Figures 2k and $\mathrm{m}$ ).

At the clinical followed up eight months after onset, and 6 weeks after termination of the final oral corticosteroid treatment, a sustained improvement of the sensory symptoms and gait is seen. At this timepoint the patient and her physiotherapist reported that the patient could walk outdoors more than 500 meters with occasional use of a single crutch. At the recent follow-up, fourthy three weeks after symptom onset the patient was able to walk and run. She had resumed working, though only at part-time. Neurological examination at this time was normal except a slight decreased sensation for touch and pain on anterolateral side of right thigh. The significant clinical improvement was confirmed by the final MRI (42 weeks after onset) which showed no residual intraspinal or nerve root lesions/contrast enhancement (Figures 2p-s).

A detail of muscle strength scores are showing in table 1. 


\section{Discussion}

Here we present a clinically challenging case of a post-infectious acute inflammatory demyelinating disease restricted to the spinal cord but also with some spinal nerve root involvement, which we believe to have been due to a spinal ADEM. The progressive phase of the disease lasted 12 weeks, a partial radiological regression was seen after immunomodulatory treatment within 24 weeks from onset of symptoms. The patient had no relapses or further deterioration of level of symptoms when seen at last follow-up eleven months after onset of disease. Despite spinal root gadolinium enhancement there were no electrophysiological signs of a polyradiculitis as judged by normal conduction velocity (NCV), but rather a central sensory disturbance reflected by pathological SSEP from the lower extremities. The normal findings on electroneurography eight weeks into the disease ruled out GBS and CIDP. Additionally, GBS was ruled even on the basis of the duration of the progressive phase of the disease exceeding 4 weeks $[2,3]$.

Acute disseminated encephalomyelitis (ADEM) is an immune mediated disease of the white matter (WM) in the brain and both white and gray matter (GM) in the spinal cord $[4,5]$. However, very little is known about ADEM confined merely to the spinal cord, and not simultaneously involving the brain. ADEM only confined to the brainstem [4] or only to the spinal cord has previously been reported [5], though the latter description is limited to a single patient. At the present time, MRI and CSF analysis are the main tools available for diagnosing ADEM [6]. The hallmarks of spinal ADEM, are abrupt onset of a multifocal spinal symptoms, gadolinium enhancement of the entire spinal cord seen on MRI, pleocytosis in the CSF and/ or a transient high immunoglobulin G index in the CSF. The disease course is usually monophasic [7], with a clinical progressive phase ranging between hours and months $[1,5,8]$, though recurrent ADEM has also been reported which in some cases may lead to a subsequent re-classification of the diagnosis as MS [8]. ADEM of cerebral and spinal type is commonly reported in children following infection or vaccination but more infrequently in adults $[6,9]$, probably due to concomitant signs of encephalopathy leading to an under-detection of spinal symptoms [7]. The detection of OCB or a persistent increase of the IgG-index in CSF indicates MS rather than ADEM. MS and ADEM could also have similar imaging findings of the brain, though ADEM is characterized by widespread white matter lesions with more poorly defined margins asymmetrically distributed supra, and infratentorially. Cerebral ADEM is accompanied with spinal cord affection in $16 \%$ of the cases [1], though there may be an underestimation of concomitant spinal ADEM due to the above-mentioned reasons. MRI of the spinal cord may show confluent intramedullary lesions with multiple areas of increased signal intensity simultaneously in the brain and the spinal cord, or merely the brain, or the spinal cord $[5,10]$. Synchronous enhancement of lesions in both the brain and spinal cord is however usually considered as a typical feature in MS, rather than ADEM. There are a number of explanations for the under-diagnosis of ADEM in general, and spinal ADEM in particular. ADEM without bloodbrain barrier damage or with an atypical pattern of the enhancement has been described [4]. However, in a complex phenotype where neurological deficit may be caused by a combined spinal and cerebral affection, the spinal component of ADEM may be under-diagnosed by clinicians only investigating the brain (and not the spinal cord) by MRI $[11,12]$. Also a significant proportion (30\%) of acute encephalitis cases are actually comprised of cerebral ADEM [10] and rarely undergo spinal imaging.

As in our case, a simultaneous peripheral nerve affection, i.e. a radiculopathy has been shown in $82 \%$ cases with ADEM $[1,10,13]$. ADEM may evolve over the course of up to 3 months [7]. Despite some differences in outcome between pediatric and adult ADEM, in most cases no new lesions are expected on MRI six months from onset of first symptoms in ADEM [14,15]. The resolution of clinical symptoms may only be partial [4].

The outcome in ADEM is as expected, dependent on response to immunomodulatory treatment given [5,7]. Corticosteroids are considered to be the most effective treatment for ADEM especially in combination with plasma exchange $[16,17]$. However, ADEM may preferentially be treated with intravenous immune globulin (IVIG), if an infection is suspected as it's etiology [12].

Our patient was transferred to a rehabilitation hospital where she began to improve during the time period that lapsed from $P E$ to the 2nd MRI fourteen weeks after onset (Figures $2 \mathrm{f}-\mathrm{i}$ ) and improved even further at the time of the $3^{\text {rd }}$ MRI (Figures $2 \mathrm{k}-\mathrm{n}$ ), twenty four weeks after onset of neurological symptoms. The MRI no. 3 revealed an almost complete resolution of earlier lesions, no new lesions and no significant medullar atrophy (Figures $2 \mathrm{k}-\mathrm{n}$ ). The patient did continue to improve radiologically (Figures 1-2) as MRI no. 4 (p-s) showed no residual lesions or contrast enhancement in the entire spinal cord. At last follow-up eleven months after onset, a sustasined post-treatment reduction in level of impairment was seen, and the patient could at this time walk independent of walking aids, and even run, even run, though with some residual painfull paresthesia in the legs.

Based on the clinical symptoms, clinical and radiological outcome after corticosteroid and PE therapy, results of MRIs and neurophysiological as well as laboratory findings, the differential diagnoses such as GBS, CIDP, MS, NMO and infectious myelitis were ruled out, leading to the final diagnosis of spinal ADEM. The following alternative causes of myelitis were also considered, but excluded:

\section{1) Transverse Myelitis (TM)}

TM which is a descriptive term simply consisting of a number of conditions where the spinal cord reacts to several pathological factors such as immune-mediated or infectious processes [7] but where no specific cause or underlying disease is found.

In this current case, there was a multilevel long spinal inflammatory lesion which is not common in TM. Furthermore, peripheral nerve root enhancement does not occur in TM.

Finally Idiopathic acute Transverse Myelitis (IATM) was not considered likely as the spinal cord lesions enhanced with gadolinium in multiple levels rather than not a single level, and since short-term out-come after immunomodulatory therapy was good, unlike what would be expected in IATM $[18,19]$.

\section{2) Neurosarcoidosis}

Neurosarcoidosis is associated with granulomatosis changes in leptomeninges, cranial nerves and hypothalamus, and it can affect the spinal cord and as well as peripheral nerves (polyneuropathy). Sarcoidosis involves the CNS in about $5-13 \%$ of cases, though involvement of the PNS is rather more common than CNS [20,21]. The most recent large case-series have shown that myelopathy can occur in up to $28 \%$ of all cases of sarcoidosis, though the incidence of neurosarcoidosis isolated merely to the spinal cord is much lower [22].

We could neither find any radiological signs of systemic sarcoidosis such as lymphadenopathy, nor an increase in blood and CSF levels of 
Table 3. Overview of major findings in conditions that present as myelitis, or mimic it.

\begin{tabular}{|c|c|c|c|c|c|c|c|c|c|c|c|}
\hline Syndrome & Study & Monophasic & Gd & Spinal lesion location & SVS & Gd-L & NCV & Rter & PE & FuW-IMD & Out-Come* \\
\hline TM & [7] & Often & Yes & Predominantly thoracolumbar & Yes & Yes & Normal & Yes & Yes & No & Improvement \\
\hline NMO & {$[27]$} & Rarely & Yes & Cervical & Yes & No & Normal & Yes & Yes & Yes & Improvement \\
\hline ADEM & {$[4,6]$} & Often & Yes & Variable & Yes & Yes & Normal & Yes & Yes & No & Recovery \\
\hline MS & [50] & Rarely & Yes & Predominantly cervical & No & No & Normal & Yes & Yes & Yes & Improvement \\
\hline CLIPPERS & {$[24,26,51]$} & Rarely & Yes & Variable & Yes & Yes & $\begin{array}{l}\text { May be } \\
\text { reduced }\end{array}$ & Yes & Yes & Yes & Improvement \\
\hline $\begin{array}{l}\text { Vitamin B12 } \\
\text { deficiency }\end{array}$ & {$[28]$} & Often & No & Predominantly cervical & Yes & No & Normal & No & No & No & No Improvement \\
\hline $\begin{array}{l}\text { Ischemic } \\
\text { myelopathy }\end{array}$ & {$[32]$} & Often & No & Predominantly thoracolumbar & No & No & Normal & No & No & No & No Improvement \\
\hline Malignancy $^{\mathrm{m}}$ & {$[33]$} & often & Yes & Variable & Yes & Yes & Normal & Yes & No & Yes & Deterioration \\
\hline Neurosarcoidosis & {$[20-23,52]$} & No & Yes & Variable & No & Yes & $\begin{array}{l}\text { May be } \\
\text { reduced }\end{array}$ & Variable & Variable & Yes & Variable \\
\hline
\end{tabular}

NCV: nerve conduction velocity; Gd: Gadolium Contrast Enhancement ; SVS: Several vertebral segments ; Gd-L: Leptomeningeal Gadolinium enhancement; Rtcr: Response To corticosteroids; PE: Plasma-Exchange; FuW-IMD: Follow-up with immunomodulatory drugs; TM: Transverse Myelitis; NMO: Neuromyelitis optica ; ADEM: Acute disseminated encephalomyelitis; MS: Multiple Sclerosis; CLIPPERS: Chronic Lymphocytic Inflammation With Pontine Perivascular Enhancement Responsive to Steroids

m: spinal cord tumors; *Out-Come 3-6 months after onset

the sarcoidosis marker ACE [23]. Additionally, the neurophysiological examination did not show any signs of polyneuropathy which would be expected as the most common PNS manifestation of sarcoidosis [22]. Finally, relapses in neurosarcoidosis are not related to antecedent infections, as was the case with our patient.

\section{3) CLIPPERS}

Chronic lymphocytic inflammation with pontine perivascular enhancement responsive to steroids (CLIPPERS) is an inflammatory condition with brainstem as well as spinal cord involvement [2426]. MRI typically shows a mass with a perivascular pattern typically most dense in the pontine and peripontine regions $[24,27,28]$. The MRI series in our case did not confirm any significant abnormity in these pathognomonic regions indicating CLIPPERS. However, the slightly increased CSF protein level and leucocytosis is a shared feature between CLIPPERS and ADEM [26]. On the other hand, the CSF flow cytometry (FACS) in our case was normal, arguing against CLIPPERS. Furthermore, a sustained clinical and radiological improvement despite withdrawal of corticosteroids is not seen in CLIPPERS [29], but was seen in our case.

\section{4) Neuromyelitis Optica (NMO)}

NMO usually shows as unilateral or bilateral optic neuritis and often with contrast enhanced edema of the optic nerve on MRI. In the current case the optic nerve were unaffected on the MRI. Furthermore, the patient did not have any increase in levels of the highly sensitive and specific marker of NMO [30], i.e. of Aquaporin-4 antibodies in the blood. In NMO the spinal cord is usually inflamed around the central canal [31] and the spinal lesions involve a length of at least 3 vertebral bodies. The radiological findings of the spinal MRI, lack of optic nerve involvement and of aquaporin-4 antibodies in blood in our patient did not support the diagnosis of NMO.

\section{5) Other causes of myelopathy}

$B_{12}$ deficiency and spinal infarction [32] were ruled out, based on the disease course, through a serological analysis of B12 and homocysteine levels as well as lack of radiological evidence of spinal ischemia. A paraneoplastic myelopathy [33] was excluded as well, due to the good response to immunotherapy and maintained clinical improvement on follow-up, the negative screen for paraneoplastic antibodies in the blood and CSF, as well as the lack of any signs of malignancy on thorax and abdominal CT (Table 3).
Finally, it is worth noting that the increase in CSF NF-Light levels has not been previously described in ADEM (Table 2). Further studies may show if the CSF NF-L levels may be used as a biomarker to followup the response to treatment and correlate to outcome in ADEM.

\section{Conclusion}

Here we describe a young adult with a post-infectious neuroinflammatory condition explicitly confined to the spinal cord, and to a lesser extent the peripheral spinal nerve roots. The investigation and follow-up pointed toward spinal ADEM (with no concomitant brain ADEM), rather than neuroinflammatory disorders such as GBS, CIDP, NMO or MS. A more careful consideration of radiological findings of isolated spinal inflammation and a systematic follow-up after administration of immunomodulatory treatment may expose a larger proportion of patients considered as having TM, to actually be suffering from spinal ADEM. The morbidity and mortality rates, as well as long-term outcome of spinal ADEM needs to be further studied.

\section{Funding information}

All authors associated with this work have not used any funding sources.

\section{Conflict of interest}

There is no conflict of interest.

\section{Patient consent}

A signed informed consent signed was obtained from the patient.

\section{References}

1. Alexander M, Murthy JM (2011) Acute disseminated encephalomyelitis: Treatment guidelines. Ann Indian Acad Neurol 14: S60-4. [Crossref]

2. Ruts L, Drenthen J, Jacobs BC, van Doorn PA; Dutch GBS Study Group (2010) Distinguishing acute-onset CIDP from fluctuating Guillain-Barre syndrome: a prospective study. Neurology 74: 1680-1686.[Crossref]

3. Asbury AK, Cornblath DR (1990) Assessment of current diagnostic criteria for Guillain-Barré syndrome. Ann Neurol 27: S21-24.[Crossref]

4. Koelman DL, Chahin S, Mar SS, Venkatesan A, Hoganson GM, et al. (2016) Acute disseminated encephalomyelitis in 228 patients: A retrospective, multicenter US study. Neurology 86: 2085-2093.[Crossref]

5. Monden Y, Yamagata T, Kuroiwa Y, Takahashi T, Mori M, et al. (2012) A case of 
ADEM with atypical MRI findings of a centrally-located long spinal cord lesion. Brain Dev 34: 380-383.[Crossref]

6. Young NP, Weinshenker BG, Lucchinetti CF (2008) Acute disseminated encephalomyelitis: current understanding and controversies. Semin Neurol 28: 84-94. [Crossref]

7. Jacob A, Weinshenker BG (2008) An approach to the diagnosis of acute transverse myelitis. Semin Neurol 28: 105-120.[Crossref]

8. Garg RK (2003) Acute disseminated encephalomyelitis. Postgraduate Medical Journal 79: $11-17$.

9. al Deeb SM, Yaqub BA, Bruyn GW, Biary NM (1997) Acute transverse myelitis. A localized form of postinfectious encephalomyelitis. Brain 120: 1115-1122. [Crossref]

10. Nasr JT, Andriola MR, Coyle PK (2000) ADEM: literature review and case report of acute psychosis presentation. Pediatr Neurol 22: 8-18.[Crossref]

11. Zaguri R, ShelefI, Ifergan G,Almog Y (2009)Fatal acute disseminated encephalomyelitis associated with cytomegalovirus infection. BMJ Case Rep. [Crossref]

12. Kanzaki A, Yabuki S (1994) [Acute disseminated encephalomyelitis (ADEM) associated with cytomegalovirus infection--a case report]. Rinsho Shinkeigaku 34: 5113. [Crossref]

13. Swamy HS, Shankar SK, Chandra PS, Aroor SR, Krishna AS, et al. (1984) Neurological complications due to beta-propiolactone (BPL)-inactivated antirabies vaccination. Clinical, electrophysiological and therapeutic aspects. J Neurol Sci 63: 111-28. [Crossref]

14. Dale RC, Branson JA (2005) Acute disseminated encephalomyelitis or multiple sclerosis: can the initial presentation help in establishing a correct diagnosis? Arch Dis Child90: 636-639. [Crossref]

15. Honkaniemi J, Dastidar P, Kähärä V, Haapasalo H (2001) Delayed MR imaging changes in acute disseminated encephalomyelitis. AJNR Am J Neuroradiol 22: 11171124.[Crossref]

16. Stricker RB, Miller RG, Kiprov DD (1992) Role of plasmapheresis in acute disseminated (postinfectious) encephalomyelitis. J Clin Apher 7: 173-9. [Crossref]

17. Kanter DS1, Horensky D, Sperling RA, Kaplan JD, Malachowski ME, et al. (1995) Plasmapheresis in fulminant acute disseminated encephalomyelitis. Neurology 45: 8247. [Crossref]

18. Krishnan C, Kaplin AI, Deshpande DM, Pardo CA, Kerr DA (2004) Transverse Myelitis: pathogenesis, diagnosis and treatment. Front Biosci 9: 1483-1499.[Crossref]

19. Cobo Calvo A, Mañé Martínez MA, Alentorn-Palau A, Bruna Escuer J, Romero Pinel L, et al. (2013) Idiopathic acute transverse myelitis: outcome and conversion to multiple sclerosis in a large series. BMC Neurol 13: 135.[Crossref]

20. Lower EE, Broderick JP, Brott TG, Baughman RP (1997) Diagnosis and management of neurological sarcoidosis. Arch Intern Med 157: 1864-1868.[Crossref]

21. Pawate S, Moses H, Sriram S (2009) Presentations and outcomes of neurosarcoidosis: a study of 54 cases. QJM 102: 449-460.[Crossref]

22. Lacomis D (2011) Neurosarcoidosis. Curr Neuropharmacol 9: 429-436.[Crossref]

23. Campbell SE, Reed CM, Bui-Mansfield LT, Fillman E (2005) Vertebral and spinal cord sarcoidosis. AJR Am J Roentgenol 184: 1686-1687.[Crossref]

24. Biotti D, Deschamps R, Shotar E, Maillart E, Obadia M, et al. (2011) CLIPPERS: chronic lymphocytic inflammation with pontine perivascular enhancement responsive to steroids. Pract Neurol 11: 349-51.

25. Keegan BM, Pittock SJ (2012) Cutting-edge questions about CLIPPERS (chronic lymphocytic inflammation with pontine perivascular enhancement responsive to steroids). Arch Neurol 69: 819-20. [Crossref]

26. Pittock SJ, Debruyne J, Krecke KN, Giannini C, van den Ameele J, et al. (2010) Chronic lymphocytic inflammation with pontine perivascular enhancement responsive to steroids (CLIPPERS). Brain 133: 2626-34. [Crossref]

27. Baharnoori M, Hohol M, Pavenski K, O'Connor P (2014) Therapeutic Effect of Plasma Exchange (PLEX) in Neuromyelitis Optica (NMO): Immediate and Long Term Response (S63.005). Neurology 82(10 Supplement).

28. Sen A, Chandrasekhar K (2013) Spinal MR imaging in Vitamin B12 deficiency: Case series; differential diagnosis of symmetrical posterior spinal cord lesions. Ann Indian Acad Neurol 16:255. [Crossref]
29. Dudesek A, Rimmele F, Tesar S, Kolbaske S, Rommer PS, et al. (2014) CLIPPERS: chronic lymphocytic inflammation with pontine perivascular enhancement responsive to steroids. Review of an increasingly recognized entity within the spectrum of inflammatory central nervous system disorders. Clin Exp Immunol175: 385-96. [Crossref]

30. Sato DK, Callegaro D, Lana-Peixoto MA, Waters PJ, de Haidar Jorge FM, et al. (2014) Distinction between MOG antibody-positive and AQP4 antibody-positive NMO spectrum disorders. Neurology 82: 474-481.[Crossref]

31. Kim HJ, Paul F, Lana-Peixoto MA, Tenembaum S, Asgari N, et al. (2015) MRI characteristics of neuromyelitis optica spectrum disorder: an international update. Neurology 84: 1165-1173.[Crossref]

32. Vargas MI, Gariani, Sztajzel R, Barnaure-Nachbar I, Delattre BM, et al. (2015) Spinal cord ischemia: practical imaging tips, pearls, and pitfalls. AJNR Am J Neuroradiol 36: 825-830.[Crossref]

33. Kennedy AM, Rogers L (1930) Spinal Cord Tumours. The Lancet 215: 854-857.

34. Matas SL, Glehn FV, Fernandes GB, Soares CA (2013) Cerebrospinal fluid analysis in the context of CNS demyelinating diseases. Arq Neuropsiquiatr 71: 685-688.[Crossref]

35. Wingerchuk DM, Lennon VA, Lucchinetti CF, Pittock SJ, Weinshenker BG (2007) The spectrum of neuromyelitis optica. Lancet Neurol 6: 805-815.[Crossref]

36. Khalil A, Mandiwanza T, Zakaria Z, Crimmins D (2016) Routine cerebrospinal fluid analysis during 'de novo' ventriculoperitoneal shunt insertion: Single Institution Experience. Br J Neurosurg 30: 427-8.[Crossref]

37. Eickhoff K, Wikström J, Poser S, Bauer H (1977) Protein profile of cerebrospinal fluid in multiple sclerosis with special reference to the function of the blood brain barrier. $J$ Neurol 214: 207-15. [Crossref]

38. Samson SK, Joseph TP, Solomon T, Daniel RT, Gnanamuthu C (2002) Spinal tumour with raised intracranial pressure. Postgrad Med $J$ 78: 628, 632-633.[Crossref]

39. Marchioni E, Tavazzi E, Minoli L, Del Blue S, Ferrante P, et al. (2008) Acute disseminated encephalomyelitis. Curr Infect Dis Rep 10: 307-314.

40. Jarius S, Paul F, Franciotta D, Ruprecht K, Ringelstein M, et al. (2011) Cerebrospinal fluid findings in aquaporin-4 antibody positive neuromyelitis optica: results from 211 lumbar punctures. J Neurol Sci306: 82-90. [Crossref]

41. Lackner P, Guengoer E, Beer R, Broessner G, Helbok R, et al. (2010) IgG-index predicts neurological morbidity in patients with infectious central nervous system diseases. BMC Infect Dis 10: 202. [Crossref]

42. (2015) Reorganized text. JAMA Otolaryngol Head Neck Surg 141: 428.

43. Jarius S, Wildemann B (2010) AQP4 antibodies in neuromyelitis optica: diagnostic and pathogenetic relevance. Nat Rev Neurol 6: 383-392.[Crossref]

44. Chen B, Chen J, House MG, Cullen KJ, Nephew KP, et al. (2012) Role of neurofilament light polypeptide in head and neck cancer chemoresistance. Mol Cancer Res 10: 305315.[Crossref]

45. Alvarez E, Piccio L, Mikesell RJ, Klawiter EC, Parks BJ, et al. (2013) CXCL13 is a biomarker of inflammation in multiple sclerosis, neuromyelitis optica, and other neurological conditions. Mult Scler 19: 1204-8. [Crossref]

46. Jiang BC, Cao DL, Zhang X, Zhang ZJ, He LN, et al. (2016) CXCL13 drives spinal astrocyte activation and neuropathic pain via CXCR5. $J$ Clin Invest 126: 745-761. [Crossref]

47. Jerrard DA, Hanna JR, Schindelheim GL (2001) Cerebrospinal fluid. J Emerg Med 21 171-178.[Crossref]

48. Jarius S, Wildemann B (2013) Aquaporin-4 antibodies, CNS acidosis and neuromyelitis optica: a potential link. Med Hypotheses 81: 1090-1095.[Crossref]

49. Twijnstra A, van Zanten AP, Hart AA, Ongerboer de Visser BW (1987) Serial lumbar and ventricle cerebrospinal fluid lactate dehydrogenase activities in patients with leptomeningeal metastases from solid and haematological tumours. J Neurol Neurosurg Psychiatry 50: 313-320. [Crossref]

50. Tumani H (2008) Corticosteroids and plasma exchange in multiple sclerosis. J Neurol 255: 36-42.[Crossref]

51. Dudesek A, Rimmele F, Tesar S, Kolbaske S, Rommer PS, et al. (2014) CLIPPERS: 
chronic lymphocytic inflammation with pontine perivascular enhancement responsive to steroids. Review of an increasingly recognized entity within the spectrum of inflammatory central nervous system disorders. Clin Exp Immunol 175: 385-396. [Crossref]
52. Olsen HH, Muratov V, Cederlund K, Lundahl J, Eklund A, et al. (2014) Therapeutic granulocyte and monocyte apheresis (GMA) for treatment refractory sarcoidosis: a pilot study of clinical effects and possible mechanisms of action. Clin Exp Immunol 177: 712-9. [Crossref]

Copyright: (C)2016 Roshanisefat H. This is an open-access article distributed under the terms of the Creative Commons Attribution License, which permits unrestricted use, distribution, and reproduction in any medium, provided the original author and source are credited. 